Review

\title{
Advances in Biophotonics Detection of Field Carcino- genesis for Colon Cancer Risk Stratification
}

\author{
Vadim Backman ${ }^{\circledR}$ and Hemant K. Roy ${ }^{2}$ \\ 1. Department of Biomedical Engineering, Northwestern University, Evanston, IL 60208, USA; \\ 2. NorthShore University HealthSystem.
}

\begin{abstract}
$\triangle$ Corresponding author: Vadim Backman, ph.: 847-491-3536 email: v-backman@northwestern.edu.
(ㅇ Ivyspring International Publisher. This is an open-access article distributed under the terms of the Creative Commons License (http://creativecommons.org/ licenses/by-nc-nd/3.0/). Reproduction is permitted for personal, noncommercial use, provided that the article is in whole, unmodified, and properly cited.
\end{abstract}

Received: 2013.01.08; Accepted: 2013.02.1I; Published: 2013.03.15

\begin{abstract}
The process of neoplastic transformation of the colon involves a progression through hyperproliferative epithelium through the aberrant crypt foci $\rightarrow$ small adenoma $\rightarrow$ large adenoma $\rightarrow$ invasive cancer $\rightarrow$ metastatic disease. These are orchestrated by sequential genetic and epigenetic events which provide the underpinnings of cellular alterations such as early induction in proliferation/suppression of apoptosis, along with the late stage increase in invasiveness. Colorectal cancer (CRC) averages 49-III mutations per tumor encompassing I0-I5 critical signaling pathways[I]. Accumulating such a high number of mutations requires a fertile mutational field, which is the hallmark of colon carcinogenesis.

While genetic susceptibility to colorectal cancer is well-known, at least half of the risk is believed to be due to exogeneous factors (e.g., obesity, diet, exercise). Understanding these risk factors represents a promising mode of tailoring screening modality and intensity. However, previous attempts using these factors (i.e., $\mathrm{NCl}$ risk calculator) have only been modestly successful with an area under receiver operating characteristics (ROC) curve (AUC) of just $0.6 \mathrm{I}$. One of the most important concepts is that risk is the interaction between these genetic and environmental components and is driven by the variety of polymorphisms. Thus, predicting risk is difficult given the complexity. On the other hand, the colonic mucosa represents the end product of the complex interplay between these multiple factors. The power of field carcinogenesis is that it reflects this interplay between genetics and environment.
\end{abstract}

Key words: Field effect, light scattering, colon cancer, LEBS, PWS, spectroscopy.

\section{Field carcinogenesis and colorectal cancer risk stratification}

There has been emerging interest in exploiting field carcinogenesis for risk stratification. Field carcinogenesis (also referred to as field effect, field defect, field of injury and field cancerization) is the concept that the genetic and environmental risk factors for colonic neoplasia provide a diffuse field of injury. It is on this "fertile" background that further stochastic genetic and epigenetic events lead to the formation of focal tumors. While the fertile mutational milieu is seen throughout the organ the focality of the lesion is a stochastic event-the particular initiating genetic/epigenetic event occurrence (generally truncation of the adenomatous polyposis coli or hypermethylation of hMLH-1 tumor suppressor genes). In other words, the field effect is one of the early steps in carcinogenesis that determines the risk of neoplastic transformation. This also implies that the genetic and environmental milieu that results in a mutation in one area of the colon should be detectable, at least in some 
form, throughout the colon.

From a clinical perspective, field carcinogenesis is well established and is the basis of clinical practice. From a synchronous lesions perspective, flexible sigmoidoscopy (examining the distal colon) has been shown to decrease the risk of not only distal but also proximal cancers[2]. Furthermore, synchronous lesions often share specific genetic mutations.

From a metachronous point of view, this is the backbone. When a patient has one colorectal neoplasia they are at higher risk of developing others elsewhere in the colon, which is exactly why more intensive colonoscopic surveillance is recommended post-polypectomy. Furthermore, the severity of the index lesion correlates with the risk of future neoplasia. Indeed, the most recent guidelines mandate colonoscopy in 3 years for advanced adenomas but only 5-10 years for smaller lesions[3].

The corollary to this is that a negative colonoscopy portends a lower risk of colorectal cancer for up to 20 years[4]. Thus, clinically speaking the negative predictive value (excluding patients from further screening) is paramount. Indeed, most of the benefit of colonoscopies comes from the first test. However, as noted, colonoscopy has a significant miss rate especially in the right (proximal) colon.

There are two main advantages of exploring field carcinogenesis for risk-stratification. The first is a matter of clinical practicality: since field carcinogenesis is not localized to a small portion of colonic mucosa only-as opposed to, for instance, dysplastic lesions - but affects colorectal mucosa diffusely, the markers of field carcinogenesis can be detected through the evaluation of the uninvolved, readily accessible mucosa as long as this mucosa is part of the field. The most convenient surrogate site is the rectal mucosa, which can be accessed without the use of an endoscope and, potentially, without bowel purge. The latter is believed to be the major reason behind patients' non-compliance with CRC screening involving endoscopic examination of the colon. Second, considering that field carcinogenesis is the fertile mutational and microenvironmental field from which focal neoplastic lesions develop following further stochastic genetic and epigenetic events, assessing field carcinogenesis lends itself to the explicit measure of CRC risk.

\section{Markers of field carcinogenesis}

As discussed above, adenomas are the classic clinical markers of field effect. (ACF $\rightarrow$ adenoma $\rightarrow$ carcinomas). These are mirrored by diffuse genetic, epigenetic, metabolomic and physiological alterations that can provide the underpinnings for biomarker development. By definition, mucosa in field carcinogenesis is histologically normal. Despite this, a variety of markers have been reported.

Micro-architectural markers include nuclear micro-morphometric parameters that are altered in the histologically normal mucosa within $5 \mathrm{~cm}$ of a tumor $[5,6]$. Karyometric analysis of microscopically normal rectal mucosa was altered in patients with neoplasia, with the magnitude correlating to the severity of proximal neoplasia[7]. Biochemical markers such as protein kinase $\mathrm{C}$ activity[8], ornithine decarboxylase[9] and mucus disaccharide content[10] from the microscopically normal mucosa in the distal mucosa have been shown to discriminate patients who harbor adenomas/carcinomas from those who are neoplasia free. Immunohistochemical markers include the loss of cytochrome $\mathrm{C}$ oxidase subunit I from the visually normal mucosa, which correlates with the presence of neoplasia elsewhere in the colon[11]. Cellular markers of proliferation[12] and apoptosis[13] in the distal mucosa predict proximal neoplasia, while the reversal of these critical early events in carcinogenesis is a good biomarker of chemoprevention. A number of genomic and epigenetic markers have been reported: microarray analysis of the histologically normal mucosa distinguished patients with and without neoplasia, with many of these differentially expressed genes implicated in the pathogenesis of neoplasia (cyclooxygenase 2, osteopontin, etc.)[14]. Importantly, the rectosigmoid (distal colon) biopsies were altered in patients harboring proximal adenomas[15] including the loss of imprinting of insulin growth factor (IGF)-2[16] and rectal mucosal TGFa[17] and IGF binding protein 3[18]. Profound proteomic aberrations in the histologically normal mucosa from neoplasia-harboring patients (both adenomas and carcinomas) have been reported[19]. Finally, microvascular alterations have been found in field carcinogenesis. While polarization-gating spectroscopy has revolutionized the ability to interrogate the colonic microvasculature[20-22], there has been other evidence including vessel corrosion casting in transitional mucosa, the expression of pro-angiogenic proteins (COX-2, iNOS, VEGF, osteopontin), the emerging concept of chemoprevention through targeting vasculature ("angioprevention") and the fact that germline vascular polymorphisms (VEGF, Eng-1) are CRC risk factors[23].

In summary, the field carcinogenesis concept is biologically robust and widely used in clinical practice. However, conventional field effect biomarkers lack the requisite performance for population screening. As detailed below, our group's work suggests that dysregulation of these diverse molecular path- 
ways may be manifested as common microarchitectural/ microvasclar consequences, making them remarkably sensitive to the field effect.

\section{Microarchitectural manifestations of field carcinogenesis}

Biomarkers can be considered as a continuum from the genetic/epigenetic, which focus on gene expression, its consequences (proteomics) and the relevant physiological correlates (metabolomics). Our group has focused on the micro/nanoscale architectural alterations.

All genetic/epigenetic consequences may have micro-architectural correlates. While it is clear that dysplasia is the hallmark of carcinogenesis, there are changes that may occur as pre-microscopically detectable abnormalities. This is because conventional microscopy is subject to the diffraction limit of light---therefore structures smaller than $\sim 250 \mathrm{~nm}$ are not detectable. Thus, while it is clear that alterations in high order chromatin structure, ribosomes, mitochondria, cytoskeleton, and other macromolecular structures are critical early events in carcinogenesis, the cells can appear microscopically normal despite the profound changes taking place.

For instance, the cytoskeleton is an integral part of the process with many of the earliest events in colon carcinogenesis interacting with structural proteins of the cytoskeleton. The adenomatous polyposis coli (APC), whose mutation is the initiating event in more than three-quarters of all CRCs, interacts with microtubules. Indeed, there have been striking changes in cytoskeleton seen in microscopically normal cells from patients with germline APC mutations (familial adenomatous polyposis). Furthermore, many other proteins in early colon cancer (e.g., c-src, E-cadherin, $\beta$-catenin) also interact with the cytoskeleton. The cytoskeleton organization may have implications for protein trafficking, mitosis, and almost any other process of cell function. Proteomic studies have suggested that treatment of the uninvolved mucosa with the secondary bile acid, deoxycholate, altered a variety of proteins including end binding (EB1), which links APC to the microtubules.

High order chromatin structure has also been implicated in early carcinogenesis. In nuclei, genomes are folded and compacted into higher-order chromatin structures, influencing gene expression, DNA replication and repair. Alterations in genome organization have been described in a variety of diseases [24, 25]. For instance, higher-order chromatin structure regulates genomic loci through partitioning into active or inactive domains. Open conformations of chromatin allow regulatory factors to interact with chromosomes while DNA bending and looping of chromatin fibers facilitates the interaction between cis-acting elements or even distantly located genome regions. Furthermore, a number of critical regulatory events such as accessibility of DNA to transcription factors may depend on the local chromatin organization[26]. Thus, chromatin remodeling may lead to a shift from a genomic homeostasis through the modulation of gene expression.

The other issue to be discussed is the stroma. There are several studies indicating that inflammatory cells in the stroma are critical. The epithelial-stromal interactions are well documented in frank tumors but there has been little known work on early alterations. There is an increasing appreciation that the inflammatory microenvironment may not only be a late manifestation and associated with metastatic potential but could also affect early events in tumorigenesis. Furthermore, there is striking evidence that stromal stiffness imbues the epithelium with more neoplastic phenotype[27]. From a molecular perspective, there is strong evidence supporting the role of lysyl oxidase (LOX) mediated stiffness in various types of carcinogenesis including CRC. Indeed, LOX appeared to be a promising therapeutic target[28]. While the nature of the stromal-epithelial interactions remains to be completely elucidated, there have been recent intriguing findings that some LOX-like enzymes deacetylate histones, serving as yet another mechanism for alterations in gene expression[29].

These changes may relate to putative morphostats originally postulated by Potter that are hypothesized to diffuse through the tissue to determine cell phenotype and maintain tissue architecture[30, 31]. For instance, modeling studies have suggested that morphostats could be a major contributor to the stromal-epithelial interaction and could potentially be responsible for initiating carcinogenesis[32].

\section{Optical techniques for detection of ultra- structural and microenvironmental alter- ations in field carcinogenesis}

Optically detectable alterations that develop in field carcinogenesis include three facets: intracellular ultrastructural, extracellular ultrastructural and microenvironmental alterations (Fig.1). We note that although the terms 'microarchitecture' and 'ultrastructure' are frequently used interchangeably, hereafter we reserve the term 'ultrastructure' in reference to the characteristics of subcellular or tissue morphology that are either below or approaching the limit of detectability of conventional microscopic histopathology. For instance, ultrastructurally abnormal 
cells may still appear to be histologically normal. Ultrastructural alterations may include changes in higher order chromatin structure, cytoskeleton, collagen fiber crosslinking, etc.

Based on the biological rationale discussed above, it follows that there would be ultrastructural and microenvironmental consequences of genomic and epigenomic events in field carcinogenesis. What are these ultrastructural and microenvironmental markers? Note that by definition uninvolved mucosa in field carcinogenesis is macroscopically (visually, endoscopically) and histologically (microscopically) normal. This implies that the detection of ultrastructural and microvascular alterations would require more sophisticated and, ideally, quantitative techniques and corresponding measures.

We first consider the quantification of microvasculature. While arteries can be visualized endoscopically, the spatial resolution limit of an endoscope ( 50-100 microns) is not sufficient to resolve small arterioles and capillaries, which is precisely where microvascular alterations are expected to take place. An accurate measurement of microvasculature on

histological sections is exceedingly difficult due to

multiple artifacts. Two techniques that are suited for the analysis of microvasculature are intravital confocal microscopy and optical spectroscopy. The power of optical spectroscopy is that hemoglobin has pathognomonic spectral absorption bands that can be used to uniquely measure parameters such as the total concentration of hemoglobin per unit volume, oxygenation (i.e., the percent of oxygenated hemoglobin relative to the total hemoglobin), and the average diameter of blood vessels. Due to the Finlay-Foster effect of saturated absorption[33], in situ optical absorption measurements are particularly sensitive to smaller blood vessels and lose sensitivity as the size increases, which makes this technique an ideal tool to assess microvasculature[34]. Another critical aspect of the measurements is depth selectivity, because microvascular alterations are the most pronounced in the pericryptal plexus of the mucosa, which supplies blood to colonocytes. A number of depth-selective optical spectroscopy techniques have been implemented to detect field carcinogenesis including polarization gating and low-coherence enhanced backscattering (LEBS) spectroscopy[20-22, 35].
(A)

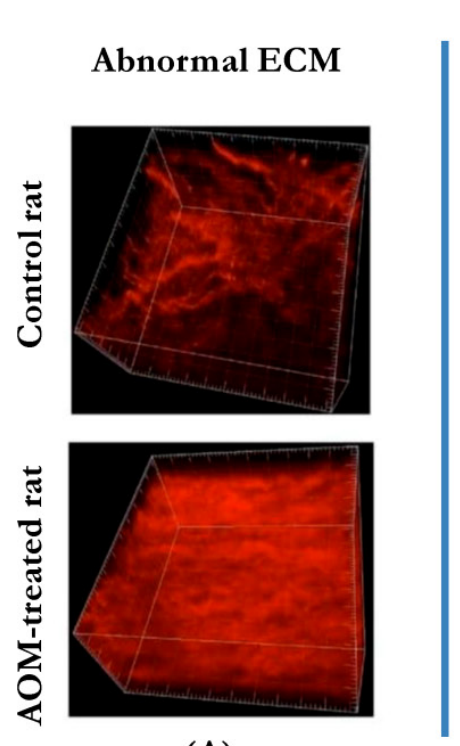

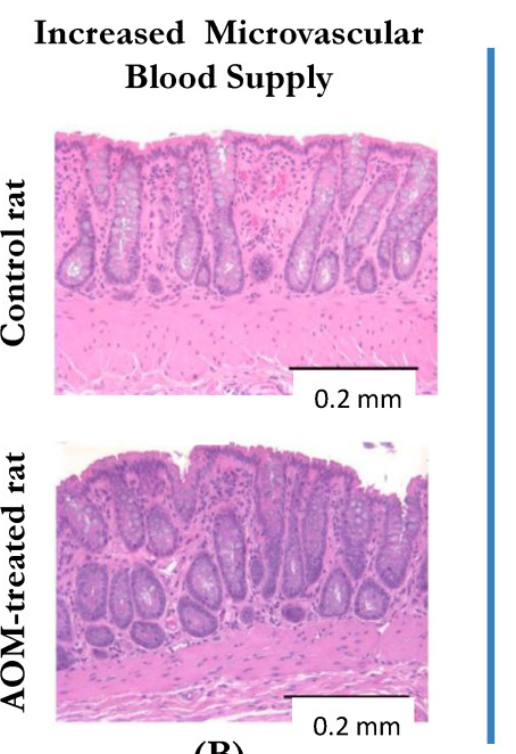

(B)
Figure I. Three manifestations of tissue alterations in colorectal field carcinogenesis: (A) Alterations in the extracellular matrix (ECM) include the abnormal cross-linking and alignment of collagen fibers. Both alterations are typical features found in the increasingly stiff matrix. Second-harmonic generation microscopy images of the collagen fiber network in the ECMs of a control rat (saline-treated, top panel) and an AOM-treated rat (bottom panel) illustrate the ECM alterations. (B): Increase in mucosal microvascular blood supply (EIBS). Paraffin embedded, hematoxylin and eosin $(\mathrm{H}$ and $\mathrm{E})$ stained colon tissue sections from the pre-neoplastic AOM-treated rat (bottom panel; 14 weeks after second AOM injection) and the age-matched control rat (top panel) show increased microvessel density in the mucosa (pericryptal network) and the superficial submucosa. No histologically-detectable dysplastic alterations in tissue were noted. Necropsies confirmed lack of evidence of neoplasia. (C): Alterations in the intracellular nanoscale architture (nanoarchitecture) include changes in higher order chromatin structure (e.g., chromatin compaction) and in the cytoplasm. The top part of the panel shows the examples of representative PWS images of three rectal cells (cells (i-iii)) from a control patient with intracellular disorder strength, which is a measure of macromolecular compaction, shown in pseudocolor. The bottom part of the panel shows three examples of PWS images of histologically normal rectal cells (cells (iv-vi)) obtained from a patient with an advanced adenoma of the colon. In the latter case, the brushing was taken from a non-neoplastic, histologically normal rectal mucosa. 


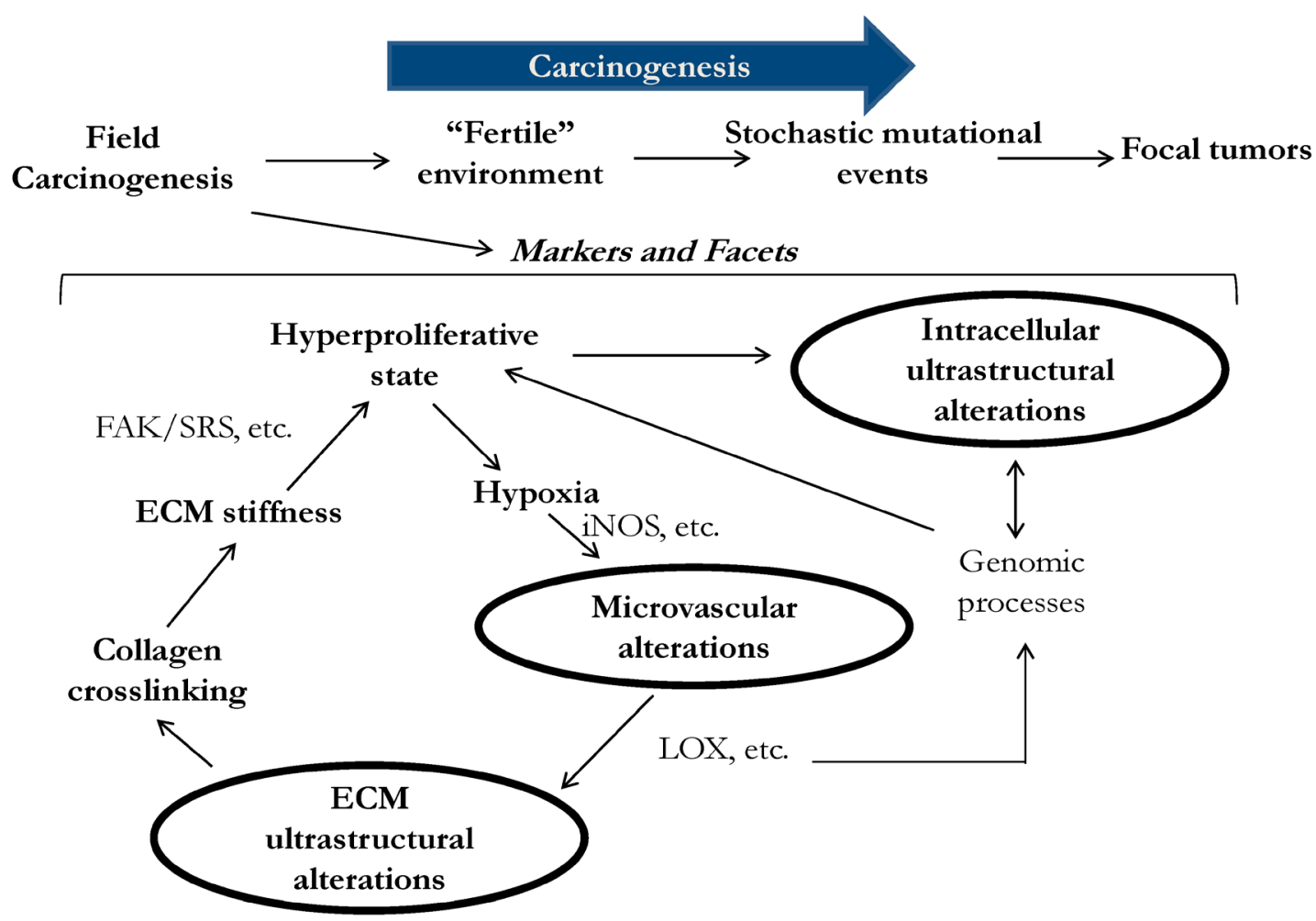

Figure 2. Three facets of microarchitectural and microvascular alterations in colorectal field carcinogenesis include changes in intracellular ultrastructure, extracellular ultrastructure and microvasculature. Early data suggests that these facets are functionally interrelated, although precise etiology and consequences of the alterations are still not fully understood.

Quantification of cellular or tissue ultrastructure requires the measurement of structures below the resolution of a microscope. As discussed above, the resolution of a conventional microscope is approximately $200 \mathrm{~nm}$ and, in most practical circumstances, $\sim 500 \mathrm{~nm}$. Smaller structures are not resolved. That is why a cell that appears to be histologically normal may still harbor ultrastructural alterations at length scales below 200-500 nanometers. One of the most comprehensive methods to quantify ultrastructure is via the correlation function of the spatial distribution of macromolecular density[36]. In particular, the functional form of the correlation function characterizes the size distribution of tissue structures. As an example, alterations such as chromatin clumping or collagen matrix cross linking change the functional form of the correlation function[37]. Tissue ultrastructure can be imaged either via high-resolution imaging such as electron microscopy or by means of light scattering. The latter approach takes advantage of the principle that although unresolvable, sub-diffractional structures can be detectable. In isolated cells, intracellular ultrastructure can be analyzed by techniques such as CLASS microscopy[38] or partial wave spectroscopic (PWS) microscopy, also re- ferred to as nanocytology[39]. In situ, tissue ultrastructure is related to the optical properties of tissue and can be measured by depth-selective light-scattering based techniques such as LEBS[40-43].

The ultrastructural and microenvironmental alterations may be present in the internal structure of colonocytes, the organization of the extracellular matrix, and microcirculation. In fact, our studies have demonstrated that all three facets are affected in field carcinogenesis (Fig. 1). This was first demonstrated in two animal models of CRC: the azoxymethane (AOM)-treated rat and the MIN-mouse[44, 45].

\section{Microvascular alterations in field carcin- ogenesis: origin and implications for risk stratification}

Whereas angiogenesis is one of the crucial events in tumor formation, increased mucosal microcirculation is a hallmark of an earlier stage of carcinogenesis, field carcinogenesis. In the AOM-treated rat model of CRC, we have shown by means of LEBS and micro-vessel density analysis (MVD) that in histologically normal colonic mucosa microvascular blood supply is increased several-fold, a phenomenon 
dubbed an early increase in microvascular blood supply (EIBS)[35, 46]. However, in contrast to 'conventional' angiogenesis, EIBS is primarily restricted to the mucosa and driven by small blood vessels such as capillaries and arterioles. EIBS is a form of neo-angiogenesis, which is most pronounced at the cryptal base approximately $200 \mathrm{um}$ into tissue. Microarray analysis showed striking dysregulation of angiogenic and anti-angiogenic factors with an overall shift in balance with increased angiogenic and decreased anti-angiogenic factors[46]. While there had been evidence from other systems that angiogenesis mediates the hyperplasia-dysplasia transition, it is becoming exceedingly clear that increased microvascular blood supply in microscopically normal colonic mucosa plays a role in at the premalignant time-point.

The consequences and etiology of EIBS in carcinogenesis are still not fully understood, although several lines of evidence indicate that mucosa in field carcinogenesis is hyperproliferative with a shift toward Warburg effect[46]. This hypoximic state induces neo-angiogenesis with inducible nitric oxide synthase (iNOS) serving as at least one of the mediators of the effect[47]. Since the concentration of proliferating colonic stem and stem-like cells is higher at the base and the lower portion of colonic crypts, this leads to EIBS being the most prominent in these tissue compartments. Thus it is likely that neo-angiogenesis in field carcinogenesis is necessary to support a hyperproliferative state in pre-dysplastic "condemned" mucosa thus allowing for the progression of focal sites toward dysplasia.

Following the animal studies, EIBS was validated in human field carcinogenesis[20]. We have developed a miniature ( $2 \mathrm{~mm}$ in diameter) fiber-optic probe. Polarization-gated optical spectroscopy was used to restrict the acquisition of light reflected from tissue to the top $100 \mathrm{um}$ (i.e., colonic mucosa). The study was conducted in 222 patients undergoing CRC-screening colonoscopy (175 with no neoplasia, 35 with non-advanced adenomas, 12 with advanced adenomas). EIBS data were acquired by the probe from 5 endoscopically normal locations in the rectum. Rectal EIBS was observed in patients harboring either advanced or multiple non-advanced adenomas anywhere in the colon. Benign colonic disease (e.g., hyperplastic polyps, diverticulosis, hemorrhoids) did not engender EIBS. Rectal EIBS was observed in patients with either distal or proximal adenomas. The performance of EIBS as a marker of carcinogenesis was assessed as the area under the receiver operating characteristic (AUC) for differentiating patients with and without advanced adenomas. AUC for a single marker, the concentration of oxygenated hemoglobin, was 0.88 . Retrospective analysis of the data showed that EIBS was not confounded by demographic and risk factors.

\section{Ultrastructural alterations in the mucosa: origin and implications for risk stratifica- tion}

In order to identify ultrastructural manifestations of field carcinogenesis, the first step was to identify the location of these changes. For this purpose we utilized a bench-top enhanced backscattering (EBS) instrument, which measured the most fundamental property characterizing light transport in tissue, light reflectance impulse response[41, 43]. We analyzed rectal biopsies from 201 subjects (96 without and 103 with colonic adenomas including 36 advanced adenomas; 2 biopsies per patient were obtained). The data showed that the difference between light scattering properties of tissue for the adenoma-harboring versus control subjects was maximized for the superficial mucosa, within the top $\sim 100-200 \mu \mathrm{m}$. These changes could potentially be due to alterations in the epithelial cells and/or the exrtacellular matrix (ECM) of the lamina propria.

In order to determine the specific origin of the ultrastructural alterations, we employed inverse scattering optical coherence tomography (ISOCT), which generates 3D images of tissue up to $\sim 300 \mu \mathrm{m}$ depth. For each resolvable voxel (approximately $10 \mathrm{x}$ $10 \times 10 \mathrm{um}$ ), ISOCT uses the spectral response of light scattering from this voxel to measure a comprehensive set of the optical properties of tissue and to characterize the spatial correlation function of macromolecular density for that particular location. The measurement was sensitive to the length scales of intra and extra-cellular structures from approximately 40 to $800 \mathrm{~nm}$. ISOCT recorded from histologically normal rectal biopsies ( $\mathrm{n}=74$ subjects including 21 with advanced adenomas) showed that the pattern of density distribution for $40-800 \mathrm{~nm}$ length scales has a mass fractal form and its fractal dimension D was increased in the mucosa in field carcinogenesis. From the optics perspective, the data showed that a key optical property, reduced scattering coefficient $\left(\mu_{s}^{\prime}\right)$, was decreased. Importantly, these alterations were found in both colonocytes and the ECM, suggesting interplay between cellular and microenvironmental events in the initial stage of carcinogenesis.

A limitation of ISOCT is that the technique, at least in its present form, cannot be implemented in vivo. In order to study the ultrastructural alterations in vivo, we developed a miniature (3.5 $\mathrm{mm}$ in diameter) LEBS fiber-optic probe[48]. The fact that the ultra- 
structural alterations in both colonocytes and ECM lead to similar optical changes makes it convenient to assess both simultaneously by means of a single LEBS reading with a penetration depth $\sim 100 \mu \mathrm{m}$. The LEBS fiber-optic probe was designed to target tissue within this optimal penetration depth. Prior to the in vivo testing of the probe, we had confirmed ex vivo that LEBS is sensitive to the ultrastructural alterations in field carcinogenesis[49]. This study was performed using a bench-top LEBS instrument in 219 subjects by acquiring LEBS readings from histologically-normal rectal biopsies obtained prior to colonoscopy. The patients were average risk with no pre-selection, resembling the makeup of the real-life screening population, including 52 with adenomas (15 advanced). LEBS validated the ultrastructural alterations that had been identified by ISOCT (an increase in D and a decrease in the reduced scattering coefficient) with the alterations present in patients with adenomas located anywhere in the colon. An LEBS marker calculated as a linear regression of LEBS parameters was progressively altered from control patients to those with 5-9 mm adenomas, and to those with advanced adenomas, thus paralleling risk. Since advanced adenomas are typically used as an endpoint to evaluate the performance of most CRC screening tests, we used this end point also. LEBS performance for discriminating patients with and without advanced adenomas was excellent (100\% sensitivity, $80 \%$ specificity, 0.90 AUC). The alterations were found in patients irrespective of the location of adenomas (distal versus proximal) and were not confounded by demographic and risk factors, which was assessed by ANCOVA and correlation analysis. Benign colonic lesions did not engender the ultrastructural alterations.

Following the ex vivo study, we set out to validate LEBS-detectable ultrastructural alterations in vivo. The study was performed by the LEBS fiber-optic probe[48] in 574 subjects. LEBS readings were recorded from the rectal mucosa prior to colonoscopy. The LEBS probe was delivered into the rectum either via an anoscope or inserted "blindly" without a visualization device and brought in contact with the mucosa. An initial set of 210 patients was used for prediction rule development, which was then prospectively validated on a new set of 346 subjects. In the validation set, the performance of the LEBS marker for predicting which subjects harbored advanced adenomas versus those who did not had $87 \%$ sensitivity and $78 \%$ specificity. Consistent with the ex vivo studies, the marker was not confounded by demographic and risk factors or benign lesions, and was altered when adenomas were present in any of the colonic segments (distal and proximal).

\section{Intracellular ultrastructural alterations: implications for risk stratification}

Whereas LEBS allows for a "single-shot" assessment of both intracellular and extracellular alterations, it is possible to specifically study intracellular events by partial wave spectroscopic (PWS) microscopy techniques, commonly known as nanocytology. In these studies, rectal colonocytes are brushed from the rectal mucosa by a conventional cyto-brush, transferred onto a glass slide, alcohol fixed and stained with a cytostain for easy visualization. By combining microscopy and spectroscopy, for each focal volume within a cell PWS measures the spectrum of light that is backscattered from this volume. By measuring the spectral variations of the backscattered light intensity PWS quantifies the heterogeneity of the spatial variations of macromolecular concentration via a parameter referred to as disorder strength (Ld), which is proportional to the amplitude and the length scale of the density variations. PWS nanocytology affords sensitivity to structures as small as $20 \mathrm{~nm}$, which is the length scale of nucleosomes, ribosomes and other macromolecular structures. In the end, PWS generates a 2D image of a cell with a value of the disorder strength measured for each topographical pixel. Following PWS imaging, an average disorder strength of a particular cellular compartment (e.g., cell nucleus), an entire cell or an ensemble of cells (e.g., cells harvested from a given patient) can be calculated. By definition, a higher value of the disorder strength corresponds to macromolecular compaction/condensation: both the length scale and the local density of macromolecular structures increase. The precise nature of the compaction depends on the intracellular location where $\mathrm{Ld}$ is increased. For example, if $\mathrm{Ld}$ is increased in the nucleus, this would correspond to chromatin condensation at the level of higher order chromatin structure.

Initial studies in cell culture, animal models, cells obtained from human colon tumors as well as histologically normal mucosa surrounding the tumor (within $4 \mathrm{~cm}$ ) showed that the disorder strength is increased in colon carcinogenesis[39, 50]. Physically, this change is consistent with the increase in D in colonocytes consistent with the ISOCT and LEBS data. The most profound Ld increase occurred in the nucleus and, to a lesser extent, in the peri-nuclear cytoplasm consistent with the alterations in the chromatin organization and rough endoplasmic reticulum[51]. In particular, in the azoxymethane (AOM)-treated rat model of colon carcinogenesis, Ld was increased in histologically normal colonocytes throughout the colon at an early pre-adenoma and pre-aberrant crypt 
foci $(\mathrm{ACF})$ time point, as early as $2-5$ weeks after AOM administration. Ld continued to progressively increase until week 10-at which point the first ACFs could be identified - after which Ld plateaued. This study confirmed that cellular nanoarchitectural alterations are some of the earliest events in carcinogenesis that precede the development of focal morphological lesions such as ACF and adenomas.

We then proceeded to test the hypothesis that an increase in Ld is an early event in carcinogenesis and, thus, a marker of field carcinogenesis in humans. The study was carried out in 146 patients[52]. Patients were recruited from those undergoing screening colonoscopy with no pre-selection. Prior to colonoscopy, rectal brushings were obtained. Based on the coefficient of inter-cellular variability of $\mathrm{Ld}$, it was estimated that for a given patient 30 cells would constitute a sufficient dataset to estimate the mean Ld with a confidence interval small compared to the effect-size between control and neoplasia-harboring patients. Accordingly, for each subject thirty randomly chosen cells were analyzed by PWS nanocytology by an operator blinded to clinical diagnosis and endoscopic findings. A patient-mean Ld was calculated for each subject.

The data demonstrated that $\mathrm{Ld}$ was increased in histologically normal cells in field carcinogenesis: although appearing normal by the criteria of microscopic histopathology, the cells possessed alterations: not in their micro-architecture which could be detectable by histology, but in the nano-architecture. The first 35 patients were used to develop a diagnostic cut off for Ld with the following 111 patients analyzed prospectively (52 control patients with no neoplasia, 34 with adenomas including 11 advanced adenomas, 12 with HNPCC (hereditary non-polyposis colorectal cancer) syndrome, and 13 with CRC). There was a progressive increase in rectal $\mathrm{Ld}$ that correlated with the potential severity of field carcinogenesis and the risk of neoplasia from patients with no neoplasia to those with non-advanced adenomas (most of which spontaneously regress) to patients with advanced adenomas (the risk of progression to CRC is $2-5 \%$ per year) to HNPCC patients (even higher, $60-80 \%$ per year, risk of progression to cancer) and finally to patients with the confirmed history of CRC. The effect was significant for all groups except for patients with diminutive adenomas, which carry a negligible risk. As a biomarker, Ld performed well with 0.90 AUC for carcinomas, 0.86 for advanced adenomas and 0.78 for all adenomas. As in case with microcirculatory and other ultrastructural alterations, Ld increase was not confounded by demographic and risk factors (e.g., age, smoking history, gender), adenomas location (distal versus proximal colon), or benign colon pathology (e.g., diverticuli, hyperplastic polyps).

\section{Biological significance of ultrastructural alterations in field carcinogenesis}

What do the ultrastructural alterations tell us about early carcinogenesis? There are three potential facets of the alterations: intracellular-these include the compaction of higher-order chromatin structure and cytoskeleton-mediated alterations-and extracellular, putatively associated with an abnormal cross-linking of ECM. As discussed above, nanocytology data revealed that some of the most pronounced alterations occur in the cell nucleus (i.e., chromatin structure). There are several lines of evidence implicating chromatin remodeling leading to chromatin compaction as an early event in carcinogenesis. Transmission electron microscopy (TEM) confirmed that chromatin is condensed in histologically normal rectal colonocytes in field carcinogenesis $(n=65)$ of patients with adenomas, colonocytes biopsied from the AOM-treated rats $(n=150)$, and the CSK-knockdown cell lines (the tumor-suppressor gene CSK-knockdown is a more aggressive cell line derived from HT29 cells, $n=40)(p<0.01)$. The length scale of the changes was $\sim 50-400 \mathrm{~nm}$, which is consistent with the range observed by the optical techniques. Chromatin de-compaction was experimentally induced by treating cells with valproic acid, which inhibits histone deacetylase (HDAC), a well-known chromatin compactor. ISOCT and PWS imaging of the valproic acid-treated cells showed a decrease in $\mathrm{D}$ and Ld.

Of note is that chromatin compaction at larger length scales (greater than $5 \mathrm{~nm}$ ) is a well-known hallmark of dysplasia - focal lesions arising on the background of field carcinogenesis - that is widely used as a marker of neoplasia in histopathology of essentially all types of cancer. The emerging picture, therefore, is that of a progressive chromatin compaction from histologically normal cells in field carcinogenesis to dysplastic cells. Evidently, chromatin clumping that is so ubiquitously observed in dysplastic cells is not unique to this stage of carcinogenesis but begins as more subtle, histologically undetectable chromatin remodeling at smaller length scales.

Another facet of intracellular ultrastructural alterations are those related to the cytoskeleton[51]. Biological implications of these changes were studied in two model systems: the AOM-treated rat model of colon cancer and HT29 \pm CSK shRNA cell lines. Not only were twenty two out of 384 cytoskeleton-regulating genes altered by real-time PCR including an early overexpression of the EB1 pro- 
to-oncogene but also the LEBS-detectable alterations and Ld increase were mitigated by treatment with a cytoskeletal inhibitor colchicine and by EB1-knockdown, thus indicating cytoskeletal abnormalities in the histologically normal cells in early carcinogenesis.

Finally, ECM alterations in field carcinogenesis (e.g., increase in D and decrease in $\mu_{s}^{\prime}$ ) were similar, albeit smaller in magnitude, to the ECM changes previously observed in tumor microenvironment[53]. Scanning electron microscopy of rectal biopsies from patients with adenomas $(n=16)$ showed increased cross-linking and local alignment of collagen fibrils/fibers, consistent with ISOCT and LEBS measurements. Furthermore, lysyl oxidase (LOX) was overexpressed in field carcinogenesis. Collagen cross-linking and the resulting increase in the mechanical stiffness of tumor stroma are well known hallmarks of tumor microenvironment with LOX being implicated as a potent collagen cross-linker[27, 54]. These mechanical and structural processes have been implicated in promoting tumor microenvironment. Again, we conclude that matrix alterations such as collagen cross-linking are not unique to tumor microenvironment but instead develop early in carcinogenesis, at the stage of field carcinogenesis.

Physiologically, the microvascular and ultrastructural changes are not completely independent but instead interconnecting facets of early carcinogenesis feeding on each other. For example, the alterations of higher order chromatin structure have been implicated and may affect a myriad of genomic processes including gene transcription and post-transcriptional modifications (Fig. 1). Indeed, a shift from a genomic homeostasis cannot occur without chromatin remodeling and thus alterations in chromatin nanoarchitecture. A greater number of hyperproliferative colonocytes that are found not only at the bottom of colonic crypts but also in what would normally be the maturation/differentiation zone of the crypts, leads to a hypoxic state, which drives neo-angiogenesis (EIBS)[46]. A change in metabolism such as the Warburg effect is another EIBS stimulus. EIBS helps support what otherwise could be an unsustainable metabolic state. Lysyl oxidase (LOX) is also driven by tissue hypoxia and leads to collagen cross-linking and increased stiffness of the stroma. Some LOX-like enzymes deacetylate histones thus directly affecting chromatin structure and gene expression. Furthermore, increased ECM stiffness promotes tumor microenvironment and has been shown to increase FAK (focal adhesion kinase)/SRC phosphorylation with the consequent activation of the signaling pathway leading to a more proliferative and invasive phenotype[55], which, as early data indicates, manifests in cellular nanoarchitectural alterations.

Clearly, there might (and expected to) be other facets of microvascular and ultrastructural alterations in early and field carcinogenesis. Although little is still known about these changes and their role in tumor formation, overall the data indicates that field carcinogenesis alterations are, in essence, subtler forms of similar alterations in the tumor, including neo-angiogenesis, chromatin remodeling (e.g., chromatin clumping) and collagen matrix cross-linking. In other words, tumor phenotype does not develop de novo but appears to already be embedded in the predisposing field carcinogenesis while the alterations are amplified and become considerably more pronounced in frankly malignant cells and tumors.

\section{Biophotonics detection of field carcino- genesis for monitoring therapeutic re- sponses}

Since optical markers represent risk, the issue arises as to whether they could represent risk modulation, too. Risk can be modified by screening. While conceptually this may be useful for responses to chemotherapy, there are few obvious clinical scenarios for this work. Lifestyle changes are just one such example. For instance, proliferation in the histologically normal mucosa has been shown to change with exercise thus supporting its role as an intermediate biomarker for risk modification. But perhaps the most promising application for field carcinogenesis biomarkers is in the monitoring of chemoprevention.

The fundamental issue in chemoprevention is personalization. Thus, only a minority of any patient population responds to a particular dosage or type of medication. For instance, aspirin is the best established chemopreventive agent. However, it only reduces risk in $30-50 \%$ of patients. Given the harms of aspirin (ulcers, bleeding, perforation), the US Preventive Services Task Force found the harms outweighed the benefits. We have demonstrated that optical markers were remarkably sensitive to the short course of the NSAID celecoxib[56]. In this regard, we have recently completed a Phase $2 b$ study which demonstrated that these markers are altered with short course aspirin therapy.

Finally, biophotonic detection of field carcinogenesis may be a platform to test novel chemopreventive agents into clinical trials. The issue is that using standard endpoints (adenomas) requires a large number of patients to be treated for protracted periods of time, which is generally prohibitively expen- 
sive. Thus finding intermediate biomarkers that can reliably and rapidly predict chemopreventive response would be critical. In this regard, we have evaluated several novel agents such as polyethylene glycol and found that the optically measured tissue fractal dimension D rapidly normalized in the MIN mouse model.

\section{Advantages of optical detection of field carcinogenesis for risk stratification}

As discussed, there are numerous potential biomarkers for field carcinogenesis. However, there are distinct advantages in assessing microarchitecture. Specifically, colorectal carcinogenesis is a molecularly heterogeneous disease with 3 distinct pathways (chromosomal instability, microsatellite instability and $\mathrm{CpG}$ island methylator phenotype). There are at least 15 molecular pathways and many of the mutations are actually passengers and low prevalence. Therefore, evaluating particular mutations has limitations. Focusing on the micro/nano-architecture has advantages in that our data suggests that these are common changes in carcinogenesis regardless of the molecular pathway involved. We have noted identical changes in MSI high disease (patients with Lynch syndrome) versus sporadic colonic neoplasia. In this regard, we have shown that alterations in a variety of proto-oncogenes/tumor suppressor genes (APC/ CSK/ EGFR/SNAIL) lead to predictable changes in cellular micro-architecture.

The other major advantage of the biophotonics approach is clinical practicality. These procedures can be done at a point of care with a fiber-optic probe or through placement of cells on slides. The number of markers is modest, suggesting the robustness of the approach to different population cohorts (gender, ethnicity, etc.).

In summary, since micro-architecture is the final common denominator for early carcinogenesis, this represents an extraordinary attractive for population screening.

\section{Acknowledgement}

This work was supported in part by grants from the National Institutes of Health R01CA128641, R01CA156186, R01CA165309, and U01 CA111257.

\section{Competing Interests}

The authors have declared that no competing interest exists.

\section{References}

1. Wood LD, Parsons DW, Jones S, Lin J, Sjoblom T, Leary RJ, et al. The genomic landscapes of human breast and colorectal cancers. Science. 2007; 318: 1108-13.

2. Schoen RE, Pinsky PF, Weissfeld JL, Yokochi LA, Church T, Laiyemo $\mathrm{AO}$, et al. Colorectal-Cancer Incidence and Mortality with Screening Flexible Sigmoidoscopy. New England Journal of Medicine. 2012; 366: 2345-57. doi:10.1056/NEJMoa1114635.

3. Lieberman DA, Weiss DG, Harford WV, Ahnen DJ, Provenzale D, Sontag SJ, et al. Five-year colon surveillance after screening colonoscopy. Gastroenterology. 2007; 133: 1077-85.

4. Singh H, Turner D, Xue L, Targownik LE, Bernstein CN. Risk of developing colorectal cancer following a negative colonoscopy examination: evidence for a 10-year interval between colonoscopies. Jama. 2006; 295: 2366-73.

5. Verhest A, Kiss R, d'Olne D, Larsimont D, Salmon I, de Launoit Y, et al. Characterization of human colorectal mucosa, polyps, and cancers by means of computerized morphonuclear image analyses. Cancer. 1990; 65: 2047-54.

6. Weyn B, Jacob W, da Silva VD, Montironi R, Hamilton PW, Thompson $\mathrm{D}$, et al. Data representation and reduction for chromatin texture in nuclei from premalignant prostatic, esophageal, and colonic lesions. Cytometry. 2000; 41: 133-8.

7. Alberts DS, Einspahr JG, Krouse RS, Prasad A, Ranger-Moore J, Hamilton P, et al. Karyometry of the colonic mucosa. Cancer Epidemiol Biomarkers Prev. 2007; 16: 2704-16.

8. McGarrity TJ, Peiffer LP. Protein kinase $\mathrm{C}$ activity as a potential marker for colorectal neoplasia. Dig Dis Sci. 1994; 39: 458-63.

9. McGarrity TJ, Peiffer LP, Bartholomew MJ, Pegg AE. Colonic polyamine content and ornithine decarboxylase activity as markers for adenomas. Cancer. 1990; 66: 1539-43.

10. Vucenik I, Gotovac J, Druzijanic N, Shamsuddin AM. Usefulness of galactose oxidase-Schiff test in rectal mucus for screening of colorectal malignancy. Anticancer Res. 2001; 21: 1247-55.

11. Payne CM, Holubec H, Bernstein C, Bernstein H, Dvorak K, Green SB, et al. Crypt-restricted loss and decreased protein expression of cytochrome C oxidase subunit I as potential hypothesis-driven biomarkers of colon cancer risk. Cancer Epidemiol Biomarkers Prev. 2005; 14: 2066-75.

12. Anti M, Marra G, Armelao F, Percesepe A, Ficarelli R, Ricciuto GM, et al. Rectal epithelial cell proliferation patterns as predictors of adenomatous colorectal polyp recurrence. Gut. 1993; 34: 525-30.

13. Bernstein C, Bernstein H, Garewal H, Dinning P, Jabi R, Sampliner RE, et al. A bile acid-induced apoptosis assay for colon cancer risk and associated quality control studies. Cancer Res. 1999; 59: 2353-7.

14. Chen L, Hao C, Chiu Y, Wong P, Melnick J, Brotman M, et al. Alteration of Gene Expression in Normal-Appearing Colon Mucosa of APCmin Mice and Human Cancer Patients. Cancer Research. 2004; 64: 3694-700.

15. Hao CY, Moore DH, Chiu YS, Wong P, Bennington JL, Smith AP, et al. Altered gene expression in normal colonic mucosa of individuals with polyps of the colon. Dis Colon Rectum. 2005; 48: 2329-35.

16. Cui H, Cruz-Correa M, Giardiello FM, Hutcheon DF, Kafonek DR, Brandenburg S, et al. Loss of IGF2 imprinting: a potential marker of colorectal cancer risk. Science. 2003; 299: 1753-5

17. Daniel CR, Bostick RM, Flanders WD, Long Q, Fedirko V, Sidelnikov E, et al. TGF-alpha expression as a potential biomarker of risk within the normal-appearing colorectal mucosa of patients with and without incident sporadic adenoma. Cancer Epidemiol Biomarkers Prev. 2009; 18: $65-73$

18. Keku TO, Sandler RS, Simmons JG, Galanko J, Woosley JT, Proffitt M, et al. Local IGFBP-3 mRNA expression, apoptosis and risk of colorectal adenomas. BMC Cancer. 2008; 8: 143.

19. Polley AC, Mulholland F, Pin C, Williams EA, Bradburn DM, Mills SJ, et al. Proteomic analysis reveals field-wide changes in protein expression in the morphologically normal mucosa of patients with colorectal neoplasia. . Cancer Res. 2006; 66: 6553-62.

20. Gomes AJ, Roy HK, Turzhitsky V, Kim Y, Rogers JD, Ruderman S, et al. Rectal Mucosal Microvascular Blood Supply Increase Is Associated with Colonic Neoplasia. Clinical Cancer Research. 2009; 15: 3110-7. doi:10.1158/1078-0432.ccr-08-2880.

21. Roy HK, Gomes AJ, Ruderman S, Bianchi LK, Goldberg MJ, Stoyneva V, et al. Optical Measurement of Rectal Microvasculature as an Adjunct to Flexible Sigmoidosocopy: Gender-Specific Implications. Cancer Prevention Research. 2010; 3: 844-51. doi:10.1158/1940-6207.capr-09-0254.

22. Roy HK, Gomes A, Turzhitsky V, Goldberg MJ, Rogers J, Ruderman S, et al. Spectroscopic microvascular blood detection from the endoscopically 
normal colonic mucosa: Biomarker for neoplasia risk. Gastroenterology. 2008; 135: 1069-78. doi:10.1053/j.gastro.2008.06.046.

23. Albini A, Noonan DM, Ferrari N. Molecular pathways for cancer angioprevention. Clinical Cancer Research. 2007; 13: 4320-5. doi:10.1158/1078-0432.ccr-07-0069.

24. Fudenberg G, Getz G, Meyerson M, Mirny LA. High order chromatin architecture shapes the landscape of chromosomal alterations in cancer. Nature Biotechnology. 2011; 29: 1109-U75. doi:10.1038/nbt.2049.

25. Michor F, Liphardt J, Ferrari M, Widom J. What does physics have to do with cancer? Nature Reviews Cancer. 2011; 11: 657-70. doi:10.1038/nrc3092.

26. Kim JS, Backman V, Szleifer I. Crowding-Induced Structural Alterations of Random-Loop Chromosome Model. Physical Review Letters. 2011;106(16):168102. doi:16810210.1103/PhysRevLett.106.168102.

27. Barker HE, Cox TR, Erler JT. The rationale for targeting the LOX family in cancer. Nature Reviews Cancer. 2012; 12: 540-52. doi:10.1038/nrc3319.

28. Barry-Hamilton V, Spangler R, Marshall D, McCauley S, Rodriguez HM, Oyasu M, et al. Allosteric inhibition of lysyl oxidase-like-2 impedes the development of a pathologic microenvironment. Nature Medicine. 2010; 16: 1009-U107. doi:10.1038/nm.2208.

29. Black JC, Whetstine JR. LOX Out, Histones: A New Enzyme Is Nipping at Your Tails. Molecular Cell. 2012; 46: 243-4. doi:10.1016/j.molcel.2012.04.023.

30. Potter JD. Morphostats: A missing concept in cancer biology. Cancer Epidemiology Biomarkers \& Prevention. 2001; 10: 161-70.

31. Potter JD. Morphogens, morphostats, microarchitecture and malignancy. Nature Reviews Cancer. 2007; 7: 464-74. doi:10.1038/nrc2146.

32. Baker SG, Soto AM, Sonnenschein C, Cappuccio A, Potter JD, Kramer BS. Plausibility of stromal initiation of epithelial cancers without a mutation in the epithelium: a computer simulation of morphostats. BMC Cancer. 2009:9:89. doi:8910.1186/1471-2407-9-89.

33. Finlay JC, Foster TH. Effect of pigment packaging on diffuse reflectance spectroscopy of samples containing red blood cells. Optics Letters. 2004; 29: 965-7. doi:10.1364/ol.29.000965.

34. Turzhitsky VM, Gomes AJ, Kim YL, Liu Y, Kromine A, Rogers JD, et al. Measuring mucosal blood supply in vivo with a polarization-gating probe. Applied Optics. 2008; 47: 6046-57.

35. Radosevich AJ, Turzhitsky VM, Mutyal NN, Rogers JD, Stoyneva V, Tiwari AK, et al. Depth-resolved measurement of mucosal microvascular blood content using low-coherence enhanced backscattering spectroscopy. Biomedical Optics Express. 2010; 1: 1196-208. doi:10.1364/boe.1.001196.

36. Rogers JD, Capoglu IR, Backman V. Nonscalar elastic light scattering from continuous random media in the Born approximation. Optics Letters. 2009; 34: 1891-3.

37. Kim JS, Pradhan P, Backman V, Szleifer I. The influence of chromosome density variations on the increase in nuclear disorder strength in carcinogenesis. Physical Biology. 2011 Feb;8(1):015004. doi:015004 10.1088/1478-3975/8/1/015004.

38. Itzkan I, Qiu L, Fang H, Zaman MM, Vitkin E, Ghiran LC, et al. Confocal light absorption and scattering spectroscopic microscopy monitors organelles in live cells with no exogenous labels. Proceedings of the National Academy of Sciences of the United States of America. 2007; 104: 17255-60. doi:10.1073/pnas.0708669104.

39. Subramanian H, Roy HK, Pradhan P, Goldberg MJ, Muldoon J, Brand RE, et al. Nanoscale Cellular Changes in Field Carcinogenesis Detected by Partial Wave Spectroscopy. Cancer Research. 2009; 69: 5357-63. doi:10.1158/0008-5472.can-08-3895.

40. Gomes AJ, Ruderman S, DelaCruz M, Wali RK, Roy HK, Backman V. In vivo measurement of the shape of the tissue-refractive-index correlation function and its application to detection of colorectal field carcinogenesis. Journal of Biomedical Optics. 2012 Apr;17(4):047005. doi:04700510.1117/1.jbo.17.4.047005.

41. Radosevich AJ, Mutyal NN, Turzhitsky V, Rogers JD, Yi J, Taflove A, et al. Measurement of the spatial backscattering impulse-response at short length scales with polarized enhanced backscattering. Optics Letters. 2011; 36: 4737-9.

42. Turzhitsky V, Radosevich AJ, Rogers JD, Mutyal NN, Backman V. Measurement of optical scattering properties with low-coherence enhanced backscattering spectroscopy. Journal of Biomedical Optics. 2011 Jun;16(6):067007. doi:06700710.1117/1.3589349.

43. Radosevich AJ, Rogers JD, Turzhitsky V, Mutyal NN, Yi J, Roy HK, et al. Polarized Enhanced Backscattering Spectroscopy for Characterization of Biological Tissues at Subdiffusion Length Scales. Ieee Journal of Selected Topics in Quantum Electronics. 2012; 18: 1313-25.

44. Roy HK, Liu Y, Wali RK, Kim YL, Kromine AK, Goldberg MJ, et al. Four-dimensional elastic light-scattering fingerprints as preneoplastic markers in the rat model of colon carcinogenesis. Gastroenterology. 2004; 126: 1071-81. doi:10.1053/j.gastro.2004.01.009.

45. Roy HK, Kim YL, Wali RK, Liu Y, Koetsier J, Kunte DP, et al. Spectral markers in preneoplastic intestinal mucosa: An accurate predictor of tumor risk in the MIN mouse. Cancer Epidemiology Biomarkers \& Prevention. 2005; 14: 1639-45.

46. Tiwari AK, Crawford SE, Radosevich A, Wali RK, Stypula Y, Kunte DP, et al. Neo-angiogenesis and the premalignant micro-circulatory augmentation of early colon carcinogenesis. Cancer Letters. 2011; 306: 205-13. doi:10.1016/j.canlet.2011.03.008.

47. Roy HK, Wali RK, Kim Y, Liu Y, Hart J, Kunte DP, et al. Inducible nitric oxide synthase (iNOS) mediates the early increase of blood supply (EIBS) in colon carcinogenesis. FEBS Letters. 2007; 581: 3857-62. doi:10.1016/j.febslet.2007.07.012.

48. Mutyal NN, Radosevich A, Gould B, Rogers JD, Gomes A, Turzhitsky V, et al. A fiber optic probe design to measure depthlimited optical properties in-vivo with Lowcoherence Enhanced Backscattering (LEBS) Spectroscopy. Optics Express. 2012; 20: 19643-57.

49. Roy HK, Turzhitsky V, Kim Y, Goldberg MJ, Watson P, Rogers JD, et al. Association between Rectal Optical Signatures and Colonic Neoplasia: Potential Applications for Screening. Cancer Research. 2009; 69: 4476-83. doi:10.1158/0008-5472.can-08-4780.

50. Subramanian H, Pradhan P, Liu Y, Capoglu IR, Li X, Rogers JD, et al. Optical methodology for detecting histologically unapparent nanoscale consequences of genetic alterations in biological cells. PNAS. 2008; 105: 20118-23. doi:10.1073/pnas.0804723105.

51. Damania D, Subramanian H, Tiwari AK, Stypula Y, Kunte D, Pradhan P, et al. Role of Cytoskeleton in Controlling the Disorder Strength of Cellular Nanoscale Architecture. Biophysical Journal. 2010; 99: 989-96. doi:10.1016/j.bpj.2010.05.023.

52. Damania D, Roy HK, Subramanian H, Weinberg DS, Rex DK, Goldberg MJ, et al. Nanocytology of Rectal Colonocytes to Assess Risk of Colon Cancer Based on Field Cancerization. Cancer Research. 2012; 72: 2720-7.

53. Nadiarnykh O, LaComb RB, Brewer MA, Campagnola PJ. Alterations of the extracellular matrix in ovarian cancer studied by Second Harmonic Generation imaging microscopy. BMC Cancer. 2010; 10: 94. doi:9410.1186/1471-2407-10-94.

54. Baker AM, Cox TR, Bird D, Lang G, Murray GI, Sun XF, et al. The Role of Lysyl Oxidase in SRC-Dependent Proliferation and Metastasis of Colorectal Cancer. Journal of the National Cancer Institute. 2011; 103: 407-24. doi:10.1093/jnci/djq569.

55. Baker A-M, Bird D, Lang G, Cox TR, Erler JT. Lysyl oxidase enzymatic function increases stiffness to drive colorectal cancer progression through FAK. Oncogene. 2012; epub.

56. Roy HK, Iversen P, Hart J, Liu Y, Koetsier JL, Kim Y, et al. Down-regulation of SNAIL suppresses MIN mouse tumorigenesis: Modulation of apoptosis proliferation, and fractal dimension. Molecular Cancer Therapeutics. 2004; 3: 1159-65. 